\title{
Methods in Mammary Gland Development and Cancer: the second ENDBC meeting - intravital imaging, genomics, modeling and metastasis
}

\author{
John Stingl', Matthew J Smalley², Marina A Glukhova ${ }^{3}$ and Mohamed Bentires-Alj*4
}

\begin{abstract}
The second meeting of the European Network for Breast Development and Cancer (ENBDC) on 'Methods in Mammary Gland Development and Cancer' was held in April 2010 in Weggis, Switzerland. The focus was on genomics and bioinformatics, extracellular matrix and stroma-epithelial cell interactions, intravital imaging, the search for metastasis founder cells and mouse models of breast cancer.
\end{abstract}

\section{Introduction}

The European Network for Breast Development and Cancer (ENBDC) organized its second meeting to foster interactions and the sharing of protocols between groups working on breast development and cancer. Graduate students, postdocs and research associates were encouraged to attend. The meeting included discussions on genomics, bioinformatics, intravital microscopy, disseminated tumor cells, ex vivo culture and in vivo models for studying breast cancer.

Bioinformatics/genomics (Chair: Matthew Smalley) Nuno Barbosa-Morais (Cancer Research UK, Cambridge Research Institute) discussed the importance of the correct annotation of microarray probes and of being sure that a probe truly maps to the gene of interest. Further problems to consider are probes that map to intron-exon boundaries, the presence of SNPs, and alternative splicing, which, as is becoming apparent, occurs on a far wider scale than previously appreciated. Splicing may lead to difficulties when summarizing data from multiple probes apparently mapping to the same

${ }^{*}$ Correspondence: Bentires@fmi.ch

${ }^{4}$ Friedrich Miescher Institute for Biomedical Research (FMI), Maulbeerstr. 66;

$\mathrm{CH}-4058$ Basel, Switzerland

Full list of author information is available at the end of the article gene but which in fact detect different splice variants. Overall, it is clear that whatever array platform is being used, application of the latest, most reliable annotation is important. In a test of annotation reliability, it was found that Refseq annotations are a more reliable guide to probe identity than GenBank/UniGene.

Britta Weigelt (Cancer Research UK, London Research Institute) spoke on the design of gene expression microarray studies, which fall into three types. The first, class comparison, is a supervised analysis to define molecular differences between predefined groups. The second, class prediction, is a supervised analysis where, after identifying the transcriptional differences between predefined groups, a genomic classifier (signature) is defined to classify new samples. It has become clear that class prediction signatures mainly identify tumors with high proliferation, although they do perform well in identifying poor prognosis tumors of the estrogen receptor-positive type. The third, class discovery, is an unbiased approach based either on unsupervised hierarchical clustering or centroids (single sample predictors (SSPs)). Both need to be used with caution. Clusters can always be detected in datasets but that does not necessarily mean they are stable or reproducible. SSPs aim to identify molecular subtypes prospectively based on the correlation between the centroids (that is, mean expression profiles for each subtype) and the tumor gene expression patterns. However, only 'basal-like' breast cancers can be robustly identified with SSPs, and for them to be introduced for routine diagnosis, the methodologies and definitions for identification of the breast cancer molecular subtypes need to be standardized.

\section{Ex vivo culture models (Chair: Marina Glukhova)}

Mohamed Bentires-Alj from the Friedrich Miescher Institute in Basel spoke on 'Ex vivo models for studying breast cancer.' He first showed that some cell biological effects are seen in three-dimensional but not in monolayer cultures, which suggests that signaling events are integrated differently in these culture models. He then described how to grow MCF10A cells as three-dimensional 
cultures in Matrigel and emphasized the importance of testing the lots of Matrigel that are suitable for MCF10A three-dimensional cultures, as MCF10A cells do not form growth-arrested acinus-like structures with some lots. Bentires-Alj's group generated three-dimensional culture models that recapitulate features of breast cancer progression, including increased proliferation, loss of polarity, decreased apoptosis, filling of the lumen and invasion of the basement membrane. Finally, he presented work from his lab on breast stem cell-niche interactions showing that three-dimensional co-cultures of human breast epithelial cells and stroma cells obtained from reduction mammoplasties in a defined culture medium allow the retention of the differentiation and proliferation potentials of breast stem/progenitor cells (S Duss, $\mathrm{H}$ Brinkhaus, and M Bentires-Alj, unpublished data).

Lone Ronnov-Jessen from the University of Copenhagen talked about 'Epithelial-stromal interactions in the human breast'. Tumor cells are known to activate the stroma; however, the stroma in turn can significantly alter gene expression in tumors cells. Comparing transcriptional profiles of breast tumor cells growing alone or in co-culture with activated breast fibroblasts, they isolated and characterized a new gene, EPSTI1 (Epithelial stromal interaction 1). EPSTI1 was not expressed in normal breast, whilst 22 of 40 breast carcinomas stained positive with anti-EPSTI1 antibody and EPSTI1 expression correlated with a lower tumor differentiation. Remarkably, ectopic expression of EPSTI1 in one human breast epithelial cell line increased tumorspheres-forming ability and induced epithelialmesenchymal transition, whereas knockdown of EPSTI1 in another cell line had the opposite effect. These findings suggest an important role for EPSTI1 in the regulation of tumor cell properties [1].

\section{Imaging and isolation of tumor cells (Chair: Mohamed Bentires-Alj)}

Jacco van Rheenen from Hubrecht Institute in Utrecht presented his work on intravital imaging of invasion and metastasis of mammary carcinomas. Intravital microscopy allows dynamic visualization of cellular and signaling events in tumors at subcellular resolution and in living animals. The cells are usually engineered to express fluorescent proteins prior to transplantation in mice and, in some cases, a photo-switchable fluorescent protein that permits tracking of the cells. To image mammary tumors, they use a mammary imaging window placed on top of the mouse mammary gland and a two-photon microscope [2]. Armed with this powerful technology, the Van Rheenen group has started to unravel new properties of tumor cells. For example, when trying to identify which cells enter the blood stream during tumor progression, they found that cells in the tumor mass enter the blood with higher efficiency than cells at the leading edge of the tumor and, thus, might be the cells that metastasize to the lung. These results warrant further investigations and may yield new insights into metastasis.

Christoph Klein from the University of Regensburg presented data on 'The search for metastasis founder cells'. The linear progression model of breast cancer postulates that normal cells progress to a fully malignant state exclusively within the primary tumor, where they develop the ability to grow into metastases. However, this view has been challenged by studies that suggest parallel evolution of cancer cells in distant sites such that cancer cells acquire successive alterations after their spread [3]. Both scenarios, however, may occur in patients. Asking when dissemination starts, the Klein group used antibodies against the epithelial marker cytokeratin to identify disseminated tumor cells (DTCs) in the bone marrow. Remarkably, the presence of a single DTC in the bone marrow of a patient with cancer accurately predicts a high risk of developing metastases. This suggests that the metastases founder cell is within the DTCs. They developed single-cell genomic analysis approaches to characterize and compare DTCs to primary tumors in humans and in animal models of cancer. They found that dissemination is often an early event and that cancer cells are not fully malignant at the time of dissemination and will not autonomously and immediately form metastases after homing to a distant site. Indeed, most of the genetic evolution of cancer cells seems to occur after dissemination. They also compared metastases to primary tumors from the same patient and found a high genomic divergence (up to $80 \%$ ) between the dominant populations in the primary tumors and metastases, further arguing for parallel evolution of cancer cells. These findings could have therapeutic relevance since patients are usually stratified for therapy based on analysis of their primary tumor and the presence of metastases. However, this may be an inappropriate way to stratify patients because the targets of adjuvant therapy are DTCs and metastases. The parallel evolution model indicates that patients should be stratified for therapy by analysis of DTCs and metastases. Finally, the results of these studies suggest that we should re-evaluate some of the cancer research models, for example, cell lines of metastatic origin (which largely exclude genomic evolution at distant sites) and preclinical models (which usually rely on the efficacy of drugs on primary tumors).

\section{Mouse models (Chair: John Stingl)}

Jos Jonkers from the Netherlands Cancer Institute in Amsterdam spoke about 'Generating and analyzing a transgenic mouse.' He discussed the pros and cons of modifying the mouse germline via transgenesis or via gene targeting in embryonic stem cells and emphasized 
the usefulness of conditional and inducible transgenics in reproducing aspects of sporadic human cancer in mice. He next presented examples of regulated in vivo knockdown using a tetracycline-inducible short hairpin RNA (shRNA) one-vector system [4]. In vivo knockdown of Bmi1 and EZH2 using this technology reproduces the phenotypes of the respective knockout mice. His group is also using this strategy to test the roles of Bmi1 and EZH2 in tumor maintenance. They cross the $K 14 \mathrm{cre} ; \mathrm{Brcal}^{\mathrm{FF}} ; \mathrm{p} 53^{\mathrm{F} / \mathrm{F}}$ and K14cre;Ecad ${ }^{F / F} ;$ F53 $3^{F / F}$ mouse mammary tumor models $[5,6]$ to mice expressing inducible shRNAs targeting Bmi1 or EZH2. An important limitation of this approach is the time consuming and expensive generation of mice with several mutations. To overcome this, Jos Jonkers discussed a more flexible approach for generating mouse models of cancer simultaneously carrying several mutations. This relies on the derivation of female embryonic stem (ES) cells from pre-existing mouse models of cancer using $3 i$ medium that effectively keeps ES cells in their ground state [7]. For example, they derived ES cells from the $K 14 c r e ; B r c a 1^{F / F} ; p 53^{F / F}$ mice and introduced an RMCE (recombinase-mediated cassette exchange) cassette into the Rosa26 locus. This allows them to introduce any cDNA or shRNA of interest directly into these ES cells and then to produce chimeric females that can be monitored for mammary tumor development. This approach will facilitate the identification of collaborating oncogenic pathways and the validation of therapeutic targets in breast cancer.

Pentao Liu from the Wellcome Trust Sanger Institute in Hinxton spoke on 'Transposons and forward genetics'. Transposons are widely used for generating mutations in model organisms. PiggyBac (PB) has recently been shown to transpose in mammalian cells after simply co-transfecting the transposon and transposase plasmids. The PB has several advantages over other DNA-transposition systems currently used in mice. First, PB transposition is very efficient and can be used to deliver various genetic elements (for example, cDNAs and small interfering RNA) to cells, including mammary epithelial cells. Second, the PB transposon carrying mutagenic cassettes can be used to perform efficient genetic screens in cultured cells or in the mouse. Third, PB transposons inserted into the genome can be excised, almost always precisely, thus allowing mutant phenotypes to be reverted. Fourth, reagents of the PB transposition system are available to the research community.

\section{Conclusion}

In the same spirit as last year [8], the meeting was an excellent occasion for brainstorming, exchanging protocols and starting new collaborations. The ENBDC network and annual meeting are now open to any lab in the world working on breast development and cancer. If you would like your lab to become a member of the ENBDC, please visit the ENBDC website [9]. Planning has already started for the 2011 meeting to be held in Weggis on April 29 to May 1, which will certainly prove as stimulating as in 2009 and 2010.

\section{Abbreviations}

DTC $=$ disseminated tumor cell; ENBDC $=$ European Network for Breast Development and Cancer; ES = embryonic stem; PB = PiggyBac; shRNA = short hairpin RNA; SSP = single sample predictor

\section{Competing interests}

The authors declare that they have no competing interests.

\section{Acknowledgements}

The ENDBC workshop was supported by the Breakthrough Breast Cancer, the Friedrich Miescher Institute for Biomedical Research (FMI) and the KGF (Kontaktgruppe für Forschungsfragen) member companies Roche, Novartis, and Merck Serono. MB-A thanks the Swiss Cancer League, the European Research Council, the Krebsliga Beider Basel and the Novartis Research Foundation for support. Work in the MS laboratory is supported by Breakthrough Breast Cancer. MS also acknowledges support from the NIHR Biomedical Research Centre. JS is funded by the The University of Cambridge, Cancer Research UK and Hutchison Whampoa Limited.

\section{Author details}

'Cancer Research UK, Cambridge Research Institute, Li Ka Shing Centre, Robinson Way, Cambridge CB2 ORE, UK. ${ }^{2}$ Breakthrough Breast Cancer Research Centre, Institute of Cancer Research, 237 Fulham Road, London SW3 6JB, UK. Institut Curie, Centre de Recherche, Centre National de la Recherche Scientifique UMR144, 26 rue d'Ulm, 75248 Paris cedex 05, France. ${ }^{4}$ Friedrich Miescher Institute for Biomedical Research (FMI), Maulbeerstr. 66; CH-4058 Basel, Switzerland.

\section{Published: 9 September 2010}

\section{References}

1. de Neergaard M, Kim J, Villadsen R, Fridriksdottir AJ, Rank F, TimmermansWielenga V, Langerod A, Borresen-Dale AL, Petersen OW, Ronnov-Jessen L: Epithelial-stromal interaction 1 (EPSTI1) substitutes for peritumoral fibroblasts in the tumor microenvironment. Am J Pathol 2010, 176:1229-1240.

2. Kedrin D, Gligorijevic B, Wyckoff J, Verkhusha WV, Condeelis J, Segall JE, van Rheenen J: Intravital imaging of metastatic behavior through a mammary imaging window. Nat Methods 2008, 5:1019-1021.

3. Klein CA: Parallel progression of primary tumours and metastases. Nat Rev Cancer 2009, 9:302-312

4. Seibler J, Kleinridders A, Kuter-Luks B, Niehaves S, Bruning JC, Schwenk F: Reversible gene knockdown in mice using a tight, inducible shRNA expression system. Nucleic Acids Res 2007, 35:e54.

5. Liu X, Holstege H, van der Gulden H, Treur-Mulder M, Zevenhoven J, Velds A, Kerkhoven RM, van Vliet MH, Wessels LF, Peterse JL, Berns A, Jonkers J: Somatic loss of BRCA1 and p53 in mice induces mammary tumors with features of human BRCA1-mutated basal-like breast cancer. Proc Natl Acad SciUSA 2007, 104:12111-12116.

6. Derksen PW, Liu X, Saridin F, van der Gulden H, Zevenhoven J, Evers B, van Beijnum JR, Griffioen AW, Vink J, Krimpenfort P, Peterse JL, Cardiff RD, Berns A, Jonkers J: Somatic inactivation of E-cadherin and p53 in mice leads to metastatic lobular mammary carcinoma through induction of anoikis resistance and angiogenesis. Cancer Cell 2006, 10:437-449.

7. Ying QL, Wray J, Nichols J, Batlle-Morera L, Doble B, Woodgett J, Cohen P, Smith $A$ : The ground state of embryonic stem cell self-renewal. Nature 2008, 453:519-523.

8. Bentires-Alj M, Clarke RB, Jonkers J, Smalley M, Stein T: It's all in the details: methods in breast development and cancer. Breast Cancer Res 2009, 11:305.

9. European Network for Breast Development and Cancer [http://www. enbdc.org/]

\section{doi: $10.1186 /$ bcr2630}

Cite this article as: Stingl J, et al.: Methods in Mammary Gland Development and Cancer: the second ENDBC meeting - intravital imaging, genomics, modeling and metastasis. Breast Cancer Research 2010, 12:311. 